\title{
A Delayed Case of Black Cohosh Associated Drug-Induced Liver Injury
}

\author{
Sara Khodor ${ }^{\mathrm{a}, \mathrm{b}}$, Max Feldman ${ }^{\mathrm{a}}$
}

\begin{abstract}
Drug-induced liver injury is the leading cause of acute liver failure in the United States. While acetaminophen is the most common culprit, there are over 1,000 prescriptions and over the counter medications that have been implicated. Black cohosh is an herbal supplement commonly used for the treatment of menopausal symptoms which has been reported to cause drug-induced liver injury. The latency period of black cohosh drug induced liver injury is highly variable which can make the diagnosis challenging. We present a case of a 59-year-old female who developed drug-induced liver injury after the use of black cohosh products.
\end{abstract}

Keywords: Black cohosh; Hepatitis; Drug-induced; Liver injury; Herbal supplement; Estroven

\section{Introduction}

Black cohosh is an over the counter herbal supplement that has been linked to various presentations of liver injury. It is important to recognize this possible association especially in the absence of other alternative causes.

\section{Case Report}

A 59-year-old female presented to the outpatient clinic after her daughter noticed her skin and eyes appeared yellow. Upon further questioning, the patient also reported dark "tea-colored" urine for the preceding 5 days. The patient otherwise felt well without complaints. She denied abdominal pain, weight loss, changes in stool, or fevers. Her current medications included levothyroxine $50 \mu \mathrm{g}$ and escitalopram $10 \mathrm{mg}$. She was previously taking over the counter (OTC) black cohosh (Estroven)

Manuscript submitted June 7, 2018, accepted June 27, 2018

aDepartment of Internal Medicine, University of South Florida, Tampa, FL, USA

bCorresponding Author: Sara Khodor, Department of Internal Medicine, University of South Florida, Tampa, FL 33620, USA.

Email: skhodor@health.usf.edu

doi: https://doi.org/10.14740/jmc3108w for vasomotor symptoms which she started 10 months prior to presentation, however she discontinued this supplement 7 weeks prior to presentation due to lack of effect. She reported one alcoholic drink every 2 weeks, took Tylenol infrequently, and had no recent travel history, sick contacts or blood transfusions. She was not sexually active, never had a tattoo, and denied a history of intravenous drug use. On exam, her vitals were normal. She was comfortable in no acute distress however appeared jaundiced with scleral icterus. Her abdomen was soft and nondistended with mild tenderness to deep palpation of the right upper quadrant. She had no stigmata of cirrhosis. She was directly admitted to the internal medicine service for further workup.

On admission, her labs were remarkable for an aspartate aminotransferase (AST) of 975, alanine transaminase (ALT) of 737 and alkaline phosphatase 311, gamma-glutamyl transferase (GGT) of 397 (normal range 9.0 - 36), total bilirubin of 14.5, international normalized ratio (INR) 1.2. CMV IgM, EBV IgM, hepatitis A IgM, hepatitis B surface antigen, hepatitis B core IgM, hepatitis $\mathrm{C}$ antibody, HIV antigen, alcohol level, acetaminophen level, antinuclear antibody (ANA), antimitochondrial antibody, anti-neutrophil cytoplasmic antibody (ANCA), anti smooth muscle, anti LKM, ceruloplasmin, and hemochromatosis DNA mutation analysis were all within normal limits. Ultrasound of right upper quadrant showed mildly heterogeneous and coarse hepatic echotexture consistent with hepatitis and cholestasis without evidence of cholelithiasis or acute cholecystitis. MRCP showed periportal edema with mild peribiliary enhancement without significant biliary ductal dilatation, no suspicious hepatic lesions. Patient underwent a CT guided (Fig. 1) liver biopsy which showed active hepatitis

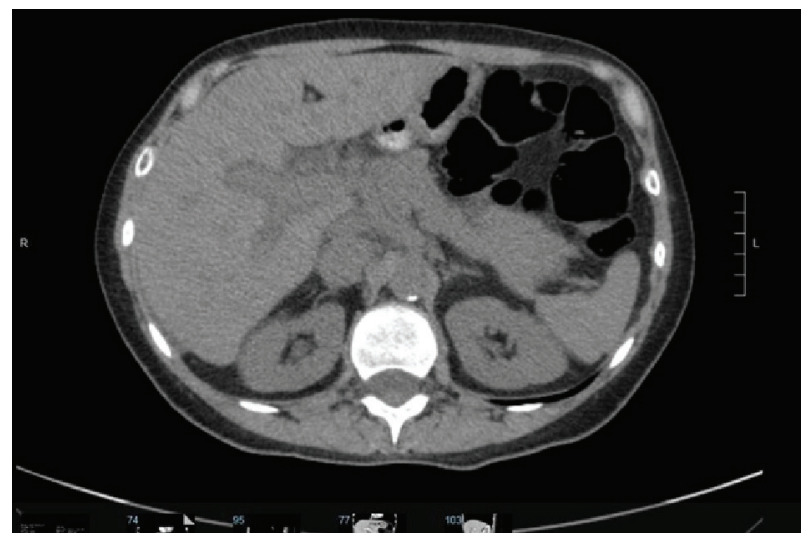

Figure 1. CT abdomen significant for peri-portal halos suggestive of periportal edema. 
with a necroinflammatory predominant pattern of injury and increased portal fibrosis with early septa formation (stage 1-2 of 4).

She was diagnosed with acute hepatitis suspected to be drug-induced liver injury (DILI) from black cohosh. Patient was instructed to avoid products containing black cohosh; she was treated supportively, and was discharged home with close follow-up. Her liver function tests improved and ultimately returned to normal 3 months post discharge.

\section{Discussion}

It is estimated that $10 \%$ of acute hepatitis cases are secondary to DILI [1] with acetaminophen being the most common culprit [2]. Other classes of medications implicated in the development of DILI include: antibiotics, antihypertensive, NSAIDs, anticonvulsants, and SSRIs. Herbal products such as black cohosh have been linked in case reports to the development of hepatotoxicity.

Black cohosh, also known as Cimicifuga racemosa, is an OTC herbal supplement that is commonly used as an antiinflammatory agent and for the treatment of postmenopausal vasomotor symptoms. To date, over 50 case reports have been published linking black cohosh to clinically apparent liver injury ranging from self-limited hepatitis, prolonged hepatitis with cholestasis, autoimmune hepatitis and acute liver failure requiring transplant [3]. The reported latency to onset of liver injury ranges from 1 to 48 weeks, but is usually within 2 to 12 weeks of starting the medication [4]. The mechanism of hepatotoxicity is suspected to be secondary to oxidative damage from accumulation of protein adducts in hepatocytes that serves as autoantigen provoking lymphocytic migration to affected areas in the liver [5].

A clear causal relationship between black cohosh and hepatotoxicity is controversial. The reason for this is multifactorial but is largely due to the fact that products labeled as black cohosh usually contain other compounds and multiple ingredients. Additionally, many reported cases often lack details in regard to temporal relation, co-medications, dosing, source, and preparation of product. An analysis of 69 cases of reported black cohosh toxicity by Teschke et al identified only one case with possible black cohosh-induced hepatotoxicity with the remaining cases labeled as having confounding variables such as pre-existing liver diseases, lack of temporal association, and use of herbal mixtures with multiple ingredients. Thus, it was concluded there was not enough evidence to substantiate a causal relationship [6].

The most likely cause of hepatotoxicity in our patient was black cohosh DILI causing hepatitis with cholestasis. We do not believe that her other medications (escitalopram, levothyroxine) contributed to her presentation as she had tolerated these medications for years prior to developing acute hepatitis and continued to take them as she improved. Furthermore, she had an extensive workup for alternative causes which was unrevealing. However, Estroven is a compound of black cohosh and many other ingredients (soy isoflavones, green tea leaf extract, yerba mate leaf extract, and magnolia bark extract) which makes a clear causal link difficult to prove.

We believe the increasing number of reports of this association warrants serious consideration and precaution. Products like black cohosh are often labeled as "all natural" which one may presume to mean "safe" and "free of side effects"; however, it is important for health care providers to understand and educate patients about this potential risk. Furthermore, we believe it is essential for healthcare providers to be aware of the variable and lengthy latency period of black cohosh liver injury, and that an exhaustive medical history including current and past herbal supplements be obtained when evaluating a patient with liver injury.

\section{Author Contributions}

Sara Khodor wrote the manuscript and is the article guarantor. Max Feldman edited the manuscript.

\section{Financial Support}

None to report.

\section{Disclosures}

Informed consent from the patient was obtained for this case report.

\section{References}

1. Zimmerman HJ. Drug-induced liver disease. Clin Liver Dis. 2000;4(1):73-96, vi.

2. Ostapowicz G, Fontana RJ, Schiodt FV, Larson A, Davern TJ, Han SH, McCashland TM, et al. Results of a prospective study of acute liver failure at 17 tertiary care centers in the United States. Ann Intern Med. 2002;137(12):947954.

3. Lim TY, Considine A, Quaglia A, Shawcross DL. Subacute liver failure secondary to black cohosh leading to liver transplantation. BMJ Case Rep. 2013;2013:bcr2013009325.

4. U.S. National Library of Medicine. Black Cohosh (Actaea Racemosa). LiverTox clinical and Research Information on Drug-Induced Liver Injury. https://livertox.nih. gov/BlackCohosh.htm. Updated 2018.

5. Enbom ET, Le MD, Oesterich L, Rutgers J, French SW. Mechanism of hepatotoxicity due to black cohosh (Cimicifuga racemosa): histological, immunohistochemical and electron microscopy analysis of two liver biopsies with clinical correlation. Exp Mol Pathol. 2014;96(3):279283.

6. Teschke R, Bahre R, Genthner A, Fuchs J, Schmidt-Taenzer W, Wolff A. Suspected black cohosh hepatotoxicity challenges and pitfalls of causality assessment. Maturitas. 2009;63(4):302-314. 